\title{
la deuxième phase du projet communautaire COSA (intercomparaison de codes de calcul géomécaniques pour le sel)
}

\author{
the second phase of the Community project COSA \\ (intercomparison of rock-mechanics computer COdes for SAlt)
}

\author{
B. CÔME \\ Commission des Communautés Européennes \\ Direction Générale "Science, Recherche et Développement »*
}

Rev. Franç. Géotech. n 51, pp. 31-40 (avril 1990)

\section{Résumé}

La deuxième phase du projet communautaire COSA (intercomparaison de codes de calcul géomécaniques pour le sel), composante du programme communautaire "Gestion et stockage des déchets radioactifs ", s'est achevée en janvier 1989. Au total, treize organismes spécialisés (dont le secrétariat technique, WS ATKINS E-S, GB) ont participé à l'exercice, qui a évalué les capacités actuelles de modélisateurs, en vue de la prévision numérique de situations thermomécaniques réelles et raisonnablement complexes. II est ainsi apparu que, bien plus que les outils numériques (codes sur ordinateur) eux-mêmes, les concepts rhéologiques pour le sel devront être l'objet de perfectionnements.

\begin{abstract}
The second phase of the COSA project (comparison of rock mechanics computer codes for salt), a component of the Community programme « Management and storage of radioactive waste ", was completed in January 1989. Thirteen specialized institutes (incl. the technical secretariat, WS ATKINS E-S, UK) participated in this exercise, which aimed at assessing the present capabilities of modellers as regards numerical blind predictions of actual, reasonably complex thermo-mechanical situations. The fact that the most substantial improvements will have to be directed towards rheological models for salt, and, to a lesser extent, to the numerical tools (computer codes) themselves, is the major conclusion of this project.
\end{abstract}

- 200, rue de la Loi - 1049 Bruxelles (B). 


\section{PRÉSENTATION GÉNÉRALE DE L'EXERCICE}

Lancée en novembre 1986, dans le cadre du troisième programme communautaire de recherche et développement sur «la gestion et le stockage des déchets radioactifs ", la deuxième phase du projet COSA a marqué l'aboutissement logique d'une progression commencée sous forme de comparaison purement numérique d'outils de calcul géomécanique (codes sur ordinateur utilisant la méthode des éléments finis) ; comme prévu à l'origine de COSA, cette deuxième phase s'est attachée à établir les capacités actuelles d'équipes spécialisées, en vue de la prévision, par le calcul, de situations thermo-mécaniques réelles, de complexité raisonnable, en étroite relation avec l'évacuation de déchets hautement radioactifs dans le sel (CÔME, 1987).

Cette deuxième phase de COSA (appelée COSA II dans la suite du texte) a maintenu sans grandes modi- fications la structure opérationnelle ayant fait ses preuves dans la première phase (LOWE et KNOWLES, 1986). Sous la coordination technique impartiale de la firme britannique WS ATKINS E-S (Epsom, Royaume-Uni), COSA II a permis la confrontation régulière des travaux d'équipes de modélisateurs et d'expérimentateurs; l'ensemble de l'exercice s'est achevé en janvier 1989 avec la publication du rapport de projet (LOWE et KNOWLES, 1989), dont le présent texte résume les points saillants.

Le tableau 1 rassemble les participants et les outils de calcul utilisés. Les traits caractéristiques de COSA ont été maintenus dans cette deuxième phase : préférence donnée au calcul a priori de problèmes réputés inconnus des participants; choix de problèmes représentatifs et $\alpha$ bien posés $*$; utilisation d'expériences existantes, d'une part pour garder une taille raisonnable au projet et, d'autre part, pour apporter une "valeur ajoutée " certaine aux expériences en question. La différence majeure d'avec COSA I est la liberté beaucoup

Tableau 1. - Participants et codes utilisés dans COSA II

Table 1. - Participants and codes used in COSA II

\begin{tabular}{|c|c|c|c|}
\hline Participants & Pays & Code thermique & Code mécanique \\
\hline $\begin{array}{l}\text { Foraky, associé au Centre d'étude } \\
\text { de l'Energie Nucléaire (CEN/SCK) }\end{array}$ & Belgique & HEAT & FLORA \\
\hline $\begin{array}{l}\text { Laboratoire du Génie Civil } \\
\text { de I'Université de Louvain-la-Neuve } \\
\text { (LGC) }\end{array}$ & Belgique & SOLVIA-TEMP & PLACRE \\
\hline $\begin{array}{l}\text { Rheinisch-Westfälische } \\
\text { Technische Hochschule (RWTH) - } \\
\text { Aachen }\end{array}$ & RFA & FAST-RZ & MAUS \\
\hline Kernforschungzentrum, Karlsruhe (KfK) & RFA & ASYTE-KA & ADINA \\
\hline $\begin{array}{l}\text { Laboratoire de Mécanique des Solides, } \\
\text { Ecole Polytechnique, Palaiseau (LMS) }\end{array}$ & France & THERM & $\begin{array}{l}\text { GEOMEC (successeur } \\
\text { d'ASTREA) }\end{array}$ \\
\hline $\begin{array}{l}\text { Département d'Etudes Mécaniques } \\
\text { et Thermiques, CEA Saclay (CEA) }\end{array}$ & France & DELFINE & INCA \\
\hline $\begin{array}{l}\text { Centre de Mécanique des Roches, } \\
\text { Ecole des Mines, Fontainebleau (EMP) }\end{array}$ & France & CHEF & $\begin{array}{l}\text { VIPLEF } \\
\text { CYSIF }\end{array}$ \\
\hline $\begin{array}{l}\text { Istituto Sperimentale } \\
\text { Modelli e Strutture, } \\
\text { Bergamo (ISMES) }\end{array}$ & Italie & GAMBLE* & GAMBLE* \\
\hline $\begin{array}{l}\text { Energieonderzoek Centrum } \\
\text { Nederlands, Petten (ECN) }\end{array}$ & Pays-Bas & ANSYS & $\begin{array}{l}\text { ANSYS } \\
\text { GOLIA }\end{array}$ \\
\hline $\begin{array}{l}\text { Empresa Nacional de Residuos } \\
\text { Radiactivos/Ecole des Mines } \\
\text { de Madrid (ENRESA) }\end{array}$ & Espagne & ADINA-T & $\begin{array}{l}\text { MINIMEF } \\
\text { ADINA }\end{array}$ \\
\hline $\begin{array}{l}\text { Gesellschaft für Strahlen und } \\
\text { Umweltforschung, Braunschweig (GSF) }\end{array}$ & RFA & \multirow{2}{*}{\multicolumn{2}{|c|}{ spécialistes du sel }} \\
\hline Technische Hogeschool, Delft & Pays-Bas & & \\
\hline
\end{tabular}

- GAMBLE est le pseudonyme d'un code à usages multiples. 
plus grande qui fut accordée aux modélisateurs quant à leur choix concernant les systèmes physiques à représenter, les conditions aux limites, la taille et le type d'éléments des maillages, les procédures d'intégration dans le temps, etc. ; pour garantir un minimum de cohérence, les spécifications des problèmes, émises par le coordinateur, comportèrent cependant toujours une partie commune à respecter par les participants.

\section{LES PROBLÈMES ÉTUDIÉS : DÉFINITION, PROCÉDURES ET DONNÉES DISPONIBLES}

\subsection{Cas étudiés}

Un survol rapide ayant montré, en 1986, le petit nombre d'expériences existantes * simples * et suffisamment proches de situations thermo-mécaniques d'évacuation dans le sel, le choix du groupe COSA se porta sur des essais réalisés entre 1979 et 1983 dans un forage profond de $300 \mathrm{~m}$, de diamètre nominal $310 \mathrm{~mm}$, réalisé par l'ECN (Pays-Bas) dans la mine de sel de Asse (RFA), en coopération avec la GSF (RFA) dans le cadre du programme communautaire (PRIJ et al., 1986). A première vue, ce choix pouvait paraître contradictoire avec une des exigences de COSA concernant l'absence de "familiarité " préalable des participants avec les cas traités; le problème fut, en fait, tourné - à la satisfaction générale - par la préparation de spécifications évitant soigneusement d'utiliser comme données d'entrée des résultats dérivant de l'expérience (interprétation de mesure in situ, etc.), et aussi par la possibilité offerte à tous les participants d'exercer leur jugement dans le cadre de variantes, à soumettre après la solution du cas "de base "décrit dans les spécifications.

D'un point de vue technique, les essais du «forage néerlandais » ainsi sélectionnés, montrent en fait une palette idéale de problèmes de complexité croissante: - une situation de convergence libre d'une section inférieure du forage à température ambiante : essai dit "IFC * (Isothermal Free Convergence) ;

- un cas de chargement d'une sonde chauffante, introduite dans le forage, par le sel environnant, venant "écraser " la sonde suite à l'échauffement: essai dit « HPP » (Heated Pressure Probe) ;

- enfin, la convergence libre d'une section de forage, mais accélérée par une sonde chauffante de diamètre inférieur : essai dit * HFCP » (Heated Free Convergence Probe).

Un schéma d'ensemble de l'arrangement est donné par la figure 1 ; chaque expérience sera détaillée plus loin (§ 3 ).

On notera au passage que ce cas constitue un exemple « idéalement simple » de problème géomécanique, celui d'un forage isolé, vertical, en massif continu homogène et (à peu près) isotrope, conditions toutes rarement satisfaites simultanément.

\subsection{Les données disponibles}

Outre les grandeurs géométriques, l'historique du forage et les caractéristiques des sondes chauffantes, les participants reçurent les résultats d'expériences de laboratoire disponibles sur échantillons du sel de Asse, soit sous forme de modèles rhéologiques déjà élaborés, soit sous forme de données expérimentales brutes (courbes de fluage, etc.), mis à disposition du projet par la GSF. En ce qui concerne les grandeurs telles que densité, coefficient de dilatation thermique, conductivité et diffusivité thermique, les valeurs sélectionnées pour COSA I furent reprises sans changement pour COSA II. Les grandeurs élastiques sont, d'après les essais de laboratoire:

$$
\begin{aligned}
& \text { Module d'Young } \mathrm{E}=24 \mathrm{GPa} \\
& \text { Coefficient de Poisson }=0,27
\end{aligned}
$$

La majorité des participants opta, dans un premier temps, pour une description du fluage en terme de fluage stationnaire, avec une dépendance de la température selon une loi d'Arrhénius, du type:

$$
\dot{\epsilon}_{e q}=A \cdot \sigma_{e q}^{n} \cdot \exp (-Q / R T)
$$

Avec :

$$
\begin{aligned}
& \mathrm{A}=0,18 / \text { jour } \\
& \mathrm{n}=5 \\
& \mathrm{Q}=54000 \mathrm{~J} / \text { mole } \\
& \mathrm{R}=\text { constante des gaz parfaits }=8,32 \mathrm{~J} / \mathrm{mol} /{ }^{\circ} \mathrm{K} \\
& \mathrm{T}=\text { température absolue en }{ }^{\circ} \mathrm{K}
\end{aligned}
$$

Pour introduire une partie de fluage primaire, certaines équipes (ex: $\mathrm{KfK}$ ) choisirent de réduire le module d'Young (ordinairement d'un facteur 2), ou bien de rajouter une équation de fluage primaire dérivée d'essais de laboratoire (ex. de la RWTH). D'autres variantes comparèrent la description du potentiel viscoplastique en terme de Von Mises ou de Tresca (ex : LMS). Enfin, le modèle de Lemaître déjà appliqué dans la phase 1 de COSA par EMP fut conservé pour COSA II, avec un raffinement supplémentaire concernant la dépendance de la température, sous forme d'un terme en :

$$
\left(a T^{2}+b T+c\right) \cdot \exp (-U / R T)
$$

a, b, c, U sont dérivés des essais existants à différentes températures.

On a d'ailleurs pu montrer que cette formulation est une expression nécessaire lorsqu'on considère une énergie d'activation fonction polynômiale de la température, et non plus simplement constante (LOWE et KNOWLES, 1989).

\section{PRINCIPAUX RÉSULTATS DE L'EXERCICE}

Comme annoncé plus haut, on se limitera ici aux principaux enseignements et à une sélection de résultats, dérivés de chaque essai modélisé. On remarquera que l'ordre de présentation est aussi l'ordre chronologique 


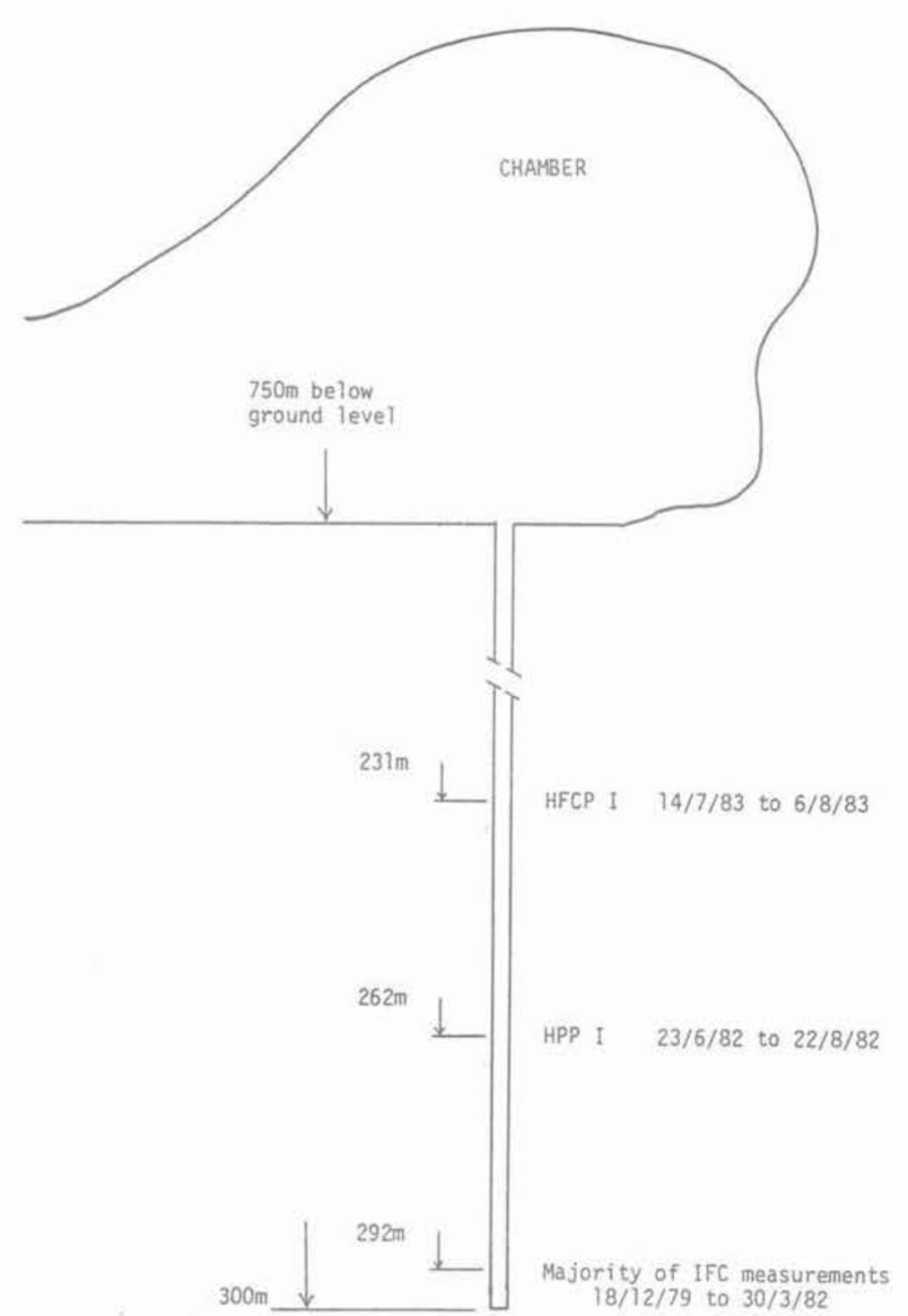

(1050m below ground)

Fig. 1. - Emplacement du « forage néerlandais » et des essais modélisés.

Fig. 1. - Location of the "Dutch hole $"$ and of the tests to be modelled.

selon lequel l'exercice fut exécuté (et probablement aussi celui de difficulté croissante des problèmes).

Pour chaque problème, il était demandé de calculer des valeurs de contraintes, déplacements (et température le cas échéant) en des points et à des dates spécifiés. On notera que la comparaison des calculs à des mesures ne pouvait être faite qu'en un nombre réduit de points (essentiellement à la paroi du forage).

\subsection{Calculs de l'essai IFC}

Les mesures de convergence libre du forage, supposé creusé instantanément, démarrèrent à la profondeur - $292 \mathrm{~m}$, trois jours après l'achèvement du forage, et furent prises en moyenne tous les $2-3$ jours pendant plus de 800 jours ; on peut donc parler ici d'expérience de longue durée. Sur la figure 2, la courbe de convergence mesurée figure en tireté (les irrégularités sont dues à un déplacement, puis remise en place, de la sonde de mesure et ne traduisent pas de mouvement saccadé du sel environnant).

Tous les participants représentèrent la zone de mesure en symétrie de révolution ( $\alpha$ tranche » plus ou moins épaisse et/ou plus ou moins étendue).

Un problème majeur fut celui du choix de l'état de contrainte initial à prendre en compte. Le " poids des terres \# théorique correspondant à la densité et à la profondeur considérée est de $22,3 \mathrm{MPa}$; cependant, certains participants inclurent la possibilité d'une décharge partielle du massif suite à l'existence même de la mine de Asse; la réduction maximale de contrainte estimée fut de $5 \mathrm{MPa}$ (valeur $\mathrm{ECN}$ ); une valeur plus faible de $2 \mathrm{MPa}$ seulement fut adoptée 


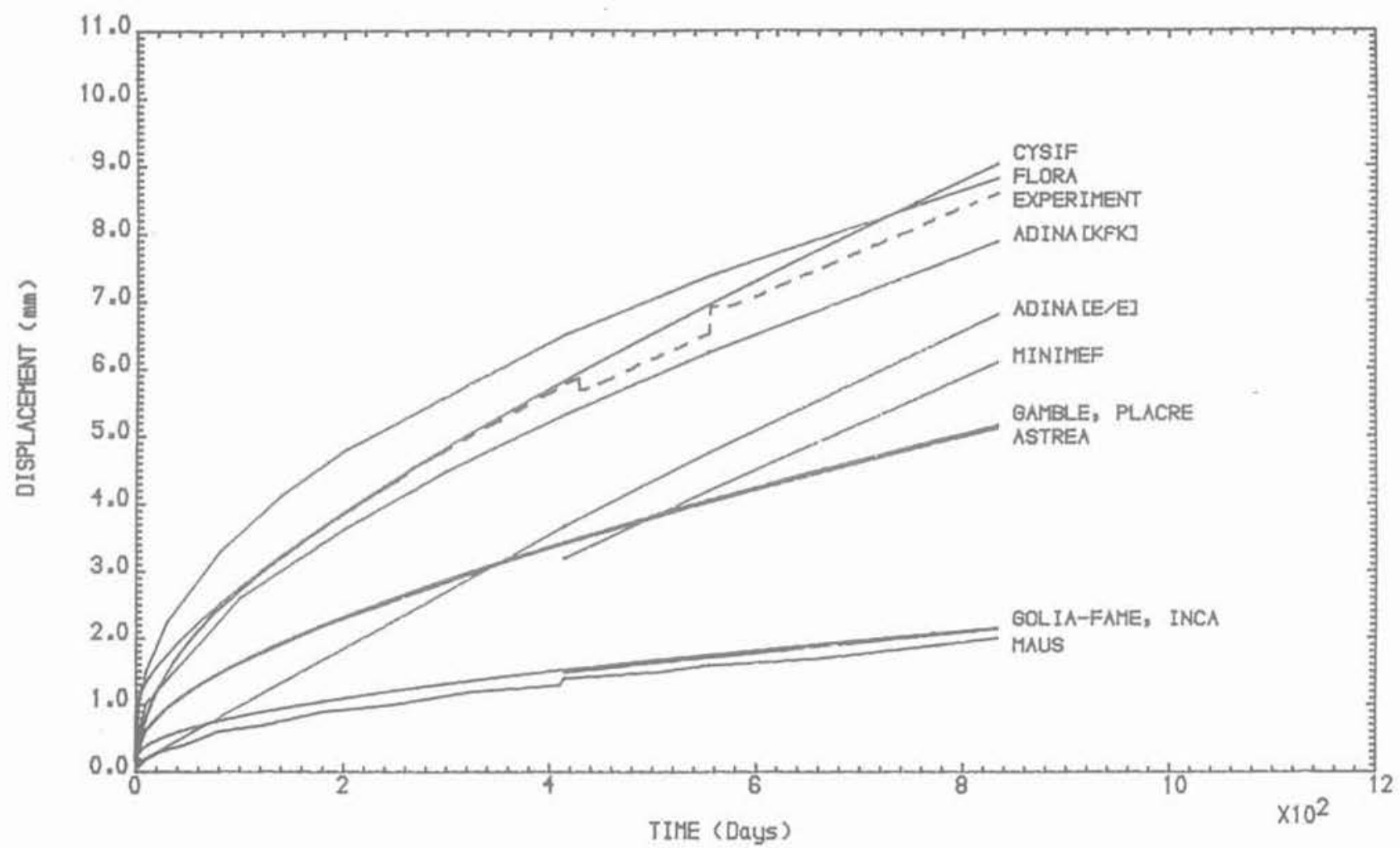

Fig. 2. - Essai «IFC ». Convergence radiale de la paroi du forage: première comparaison de la mesure (trait interrompu) et des calculs (traits pleins).

Fig. 2. - aIFC N experiment. Radial convergence of borehole wall: first comparison between measurements (dashed line) and calculations (solid lines)

par KfK suite à une étude par éléments finis indépendante de COSA mais mise à la disposition des participants.

La figure 2 rassemble les résultats de calculs, au niveau - $292 \mathrm{~m}$, de convergence " a priori ", c'està-dire selon les idées initiales des modélisateurs. On peut les classer en trois types:

- les valeurs de convergence «faibles » sont celles obtenues pour un état de contrainte initial réduit, et l'hypothèse du fluage secondaire seul;

- les convergences «moyennes " associent en général l'hypothèse de fluage secondaire seul et un état de contrainte initial sans réduction ;

- les valeurs les plus voisines de l'expérience sont obtenues pour l'état de contrainte sans réduction et un fluage secondaire/module d'Young " modifiés », ou directement - fait notable - par le modèle de Lemaitre.

Un grand nombre d'autres variantes permettent de raffiner encore les conclusions précédentes. En outre, une intéressante vérification de la justesse numérique des calculs peut aussi être réalisée sur cet exercice, à propos des codes décrivant le fluage en termes de fluage secondaire uniquement.

On montre en effet (PRIJ et al., 1986) qu'on peut, dans ces conditions, « normaliser » les courbes de convergence en utilisant les facteurs suivants :

convergence normalisée $=\frac{\text { convergence mesurée }}{\text { convergence élastique }}$ avec convergence élastique $=\frac{3 \mathrm{P} \mathrm{r}}{2 \mathrm{E}}$

$\mathrm{P}=$ pression (contrainte lithostatique) initiale

$\mathrm{r}=$ rayon du forage

$E$ = module d'Young

(matériau supposé incompressible)

temps normalisé $=\frac{\text { temps réel }}{\text { temps caractéristique }}$

avec temps caractéristique $=$

$$
(P \sqrt{3})^{1-n} /(E \cdot A \cdot \exp (-Q / R T))
$$

$\mathrm{A}, \mathrm{n}, \mathrm{Q}$ : paramètres de la loi de fluage secondaire.

Les résultats de cette normalisation sont reportés sur la figure 3 , qui permet de confirmer l'exactitude numérique des calculs (toute erreur se traduit par un écart notable par rapport à la courbe «commune »). Il s'agit donc là d'une vérification supplémentaire des outils de calcul.

\subsection{Calculs de l'essai HPP}

Pour cette expérience, une sonde métallique chauffante, tube de diamètre $296 \mathrm{~mm}$ et de hauteur $6 \mathrm{~m}$ (dont $3 \mathrm{~m}$ de partie chauffante centrale), fut descendue 


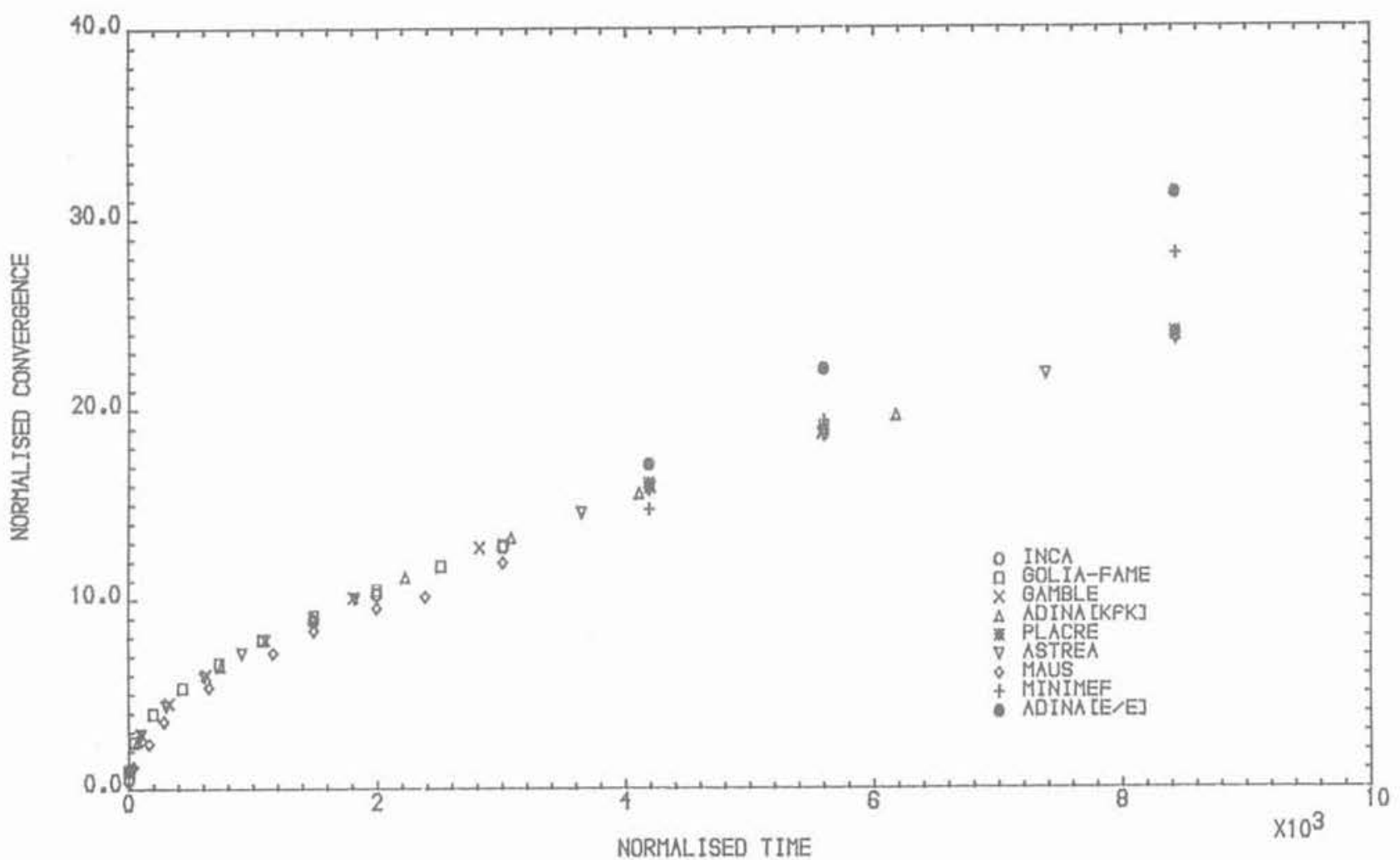

Fig. 3. - Essai "IFC ". Convergence normalisée, calculable dans l'hypothèse du fluage stationnaire seulement. Fig. 3. - "IFC "experiment. Normalized convergence curve, which can be calculated only with the hypothesis of stationary creep.

dans le forage jusqu'à ce qu'elle se "coince "à la profondeur $262 \mathrm{~m}$; le contact avec le sel n'était probablement pas uniforme sur toute sa surface, mais plus vraisemblablement obtenu par quelques aspérités de la paroi. Le problème spécifia cependant un contact initial parfait entre sel et métal de la sonde. Entre juin et août 1982, soit sur 60 jours, une puissance électrique à peu près constante de $4715 \mathrm{~W}$ fut injectée dans la sonde; la pression résultante du sel chauffé fut relevée par la déformation de la sonde (au moyen de 23 capteurs de déformations solidaires de celle-ci), et la température de la sonde et du sel (à l'interface) fut mesurée par une cinquantaine de thermocouples.

Comme l'essai avait eu lieu près de deux ans et demi après la réalisation du sondage, il était souhaitable de prendre comme condition initiale du calcul thermomécanique l'état de contrainte obtenu après cette période de convergence libre.

\subsubsection{Calculs thermiques}

A titre d'exemple, la figure 4 regroupe les calculs de la température au contact sel-sonde, au milieu de celle-ci ; il est frappant de constater l'accord entre ces différents résultats numériques, mais aussi leur désaccord notable d'avec la mesure en place (calcul: en moyenne $220^{\circ} \mathrm{C}$; mesure : $184^{\circ} \mathrm{C}$, soit un écart de $20 \%$ ). Les discussions n'ont cependant pas permis d'identifier avec certitude la ou les raisons de la différence : soit des propriétés thermiques réelles du sel différentes de celles adoptées pour le calcul, soit des pertes thermiques (par les câbles reliant la sonde à la surface, par exemple), soit les deux. On notera qu'à $10 \mathrm{~m}$ de la sonde, l'échauffement (calculé) dans le sel n'excède pas $1^{\circ} \mathrm{C}$.

\subsubsection{Calculs mécaniques}

Comme pour l'essai IFC, une vaste gamme d'hypothèses sur le sel fut utilisée par les divers modélisateurs et se reflète dans les résultats calculés (noter que les spécifications de la sonde, incluses dans le modèle, furent communes à toutes les équipes).

De nouveau à titre d'exemple, on donne en figure 5 les premières * prévisions * de l'effort normal (compression) appliqué par le sel dans le plan médian de la sonde. Malgré une reproduction correcte da la tendance (évolution chronologique) du phénomène, il existe là encore une dispersion notable des résultats calculés. Un examen attentif de ces résultats, et des variantes soumises ultérieurement, montre que la pression du sel sur la sonde est gouvernée par la réponse élastique initiale du sel à la répartition de température. Le sel chauffé, localisé, est fortement confiné par son contact initial avec la sonde, et aussi par l'énorme masse de sel froid qui l'environne. C'est ainsi que la pression mise en jeu dépend de l'échauffement, du coefficient de dilatation thermique et du module d'Young du sel. Il n'est donc pas surprenant que la pression calculée excède la pression mesurée, car la température calculée est supérieure à la température effectivement mesurée. 


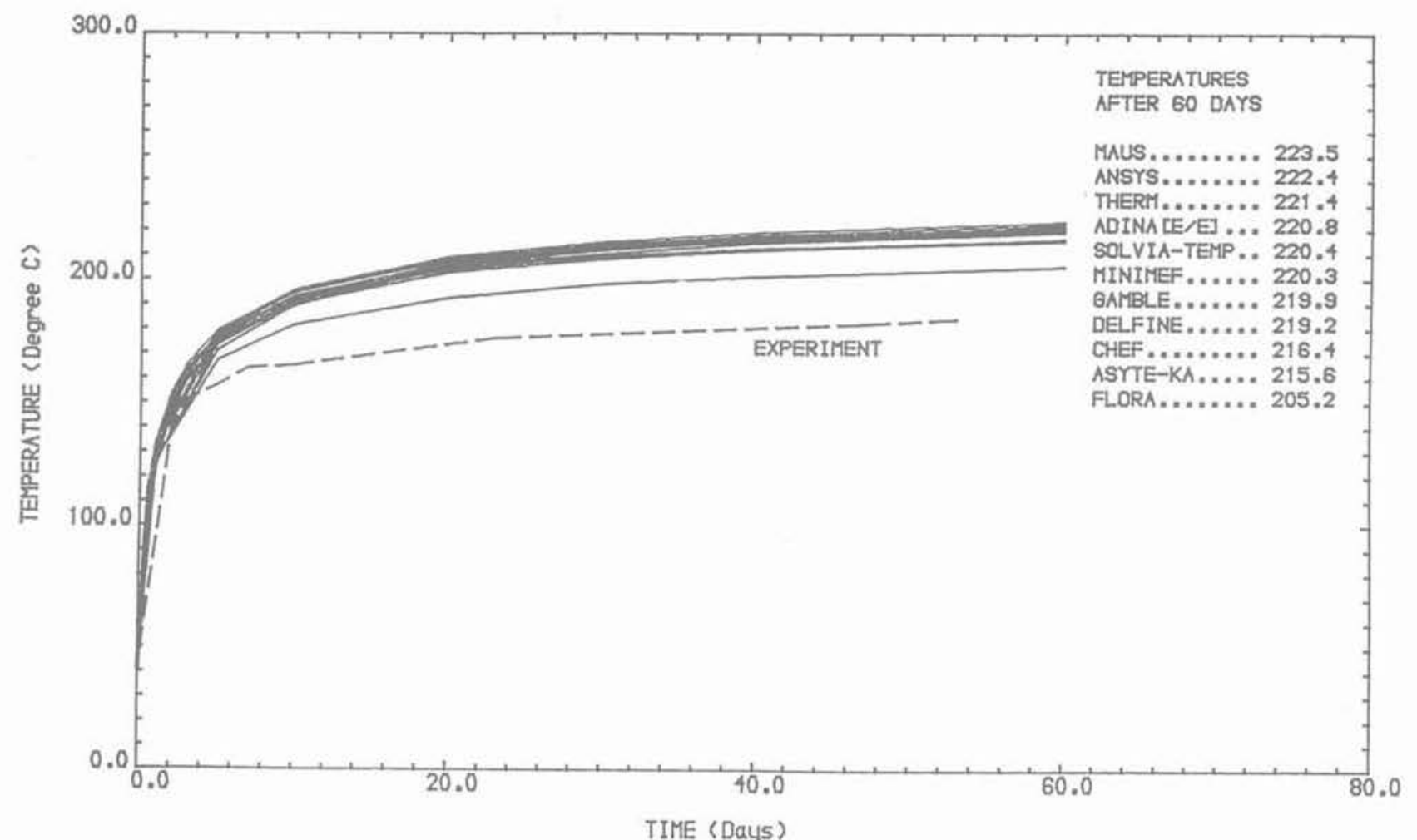

Fig. 4. - Essai « HPP n. Température à la paroi du forage : première comparaison de la mesure (trait interrompu) et des calculs (traits pleins).

Fig. 4. - "HPP experiment. Temperature at borehole wall: first comparison between measurement (dashed line) and calculations (solid lines).

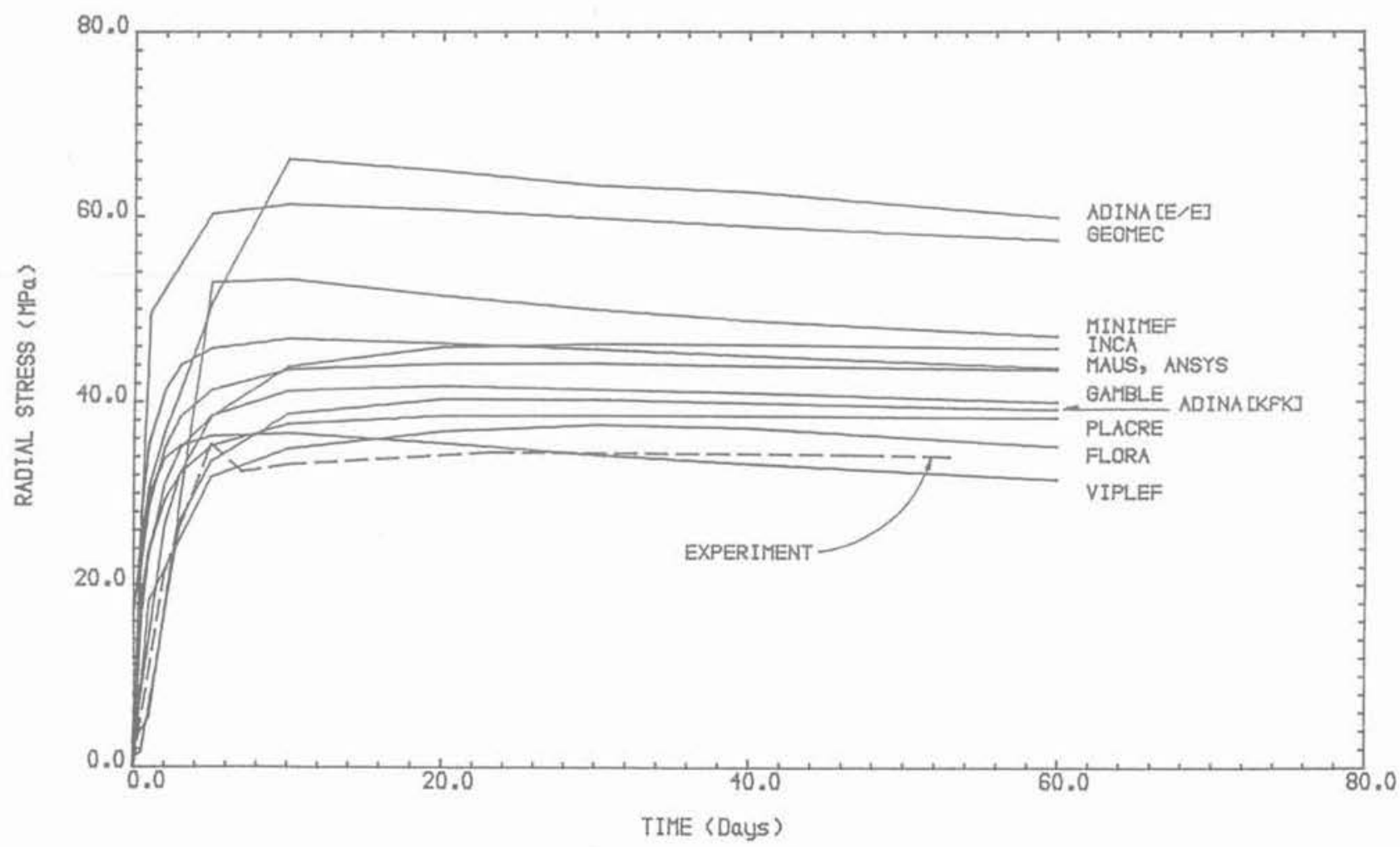

Fig. 5. - Essai « HPP „. Pression radiale exercée sur la sonde: première comparaison de la mesure (trait interrompu) et des calculs (traits pleins).

Fig. 5. - "HPP " experiment. Radial pressure exerted on the probe : first comparison between measurement (dashed line) and calculations (solid lines). 
Par contre, la description du fluage, et la valeur de la contrainte lithostatique d'origine, semblent avoir une influence beaucoup moins forte que dans le calcul IFC. Enfin, certains participants (dont KfK) ont testé l'influence d'un vide initial entre le sel et la sonde (typiquement, entre 1 et 2 millimètres). Contrairement à ce qu'on pourrait supposer, l'introduction de ce vide annulaire initial n'influence que peu le résultat.

On notera pour finir l'importance des contraintes engendrées : près de $35 \mathrm{MPa}$ appliqués sur la sonde, pour environ $22 \mathrm{MPa}$ de pression lithostatique initiale à cette cote.

\subsection{Calculs de l'essai HFCP}

Dans cet essai, une sonde chauffante (longueur $6 \mathrm{~m}$ dont $3 \mathrm{~m}$ de partie chauffante, diamètre extérieur 166 $\mathrm{mm}$ ) fut descendue dans le forage à la cote - $231 \mathrm{~m}$ (soit environ $30 \mathrm{~m}$ au-dessus de la sonde HPP). La sonde portait des palpeurs fonctionnant selon le principe du * caliper *, permettant ainsi de contrôler la valeur de l'annulaire entre paroi et sonde 1305 jours après la foration du sondage (soit en juillet 1983), une puissance électrique constante de $6000 \mathrm{~W}$ fut injectée dans la sonde; des pertes diverses réduisirent la valeur efficace à $5142 \mathrm{~W}$. Au bout de 19 jours de chauffage, l'annulaire de $65 \mathrm{~mm}$ environ s'était refermé sur la sonde et le chauffage fut arrêté; les mesures furent continuées pendant 3 autres jours, puis on retira la sonde avant qu'elle ne soit piégée par le sel. L'analyse thermique fut demandée pour la totalité de l'essai (incluant la phase de refroidissement) ; pour maintenir l'exercice à un niveau de complexité raisonnable, seule la phase de chauffage fit l'objet de lanalyse mécanique. A la cote considérée, la contrainte initiale lithostatique pouvait être estimée à 21 $\mathrm{MPa}$, la convergence libre du forage affectant bien sûr cette valeur.

\subsubsection{Calculs thermiques}

La difficulté principale fut, dans ce cas, le transfert de chaleur de la sonde au sel par radiation; des facteurs d'émission de 0,95 et 0,4 furent recommandés respectivement pour le sel et le métal de la sonde. Certains participants firent varier le diamètre du forage, au cours de l'essai, dans le calcul thermique lui-même.

Les températures calculées et mesurées à la paroi du forage, ainsi qu'une estimation de la température de la sonde elle-même, sont données sur la figure 6 . Il faut noter que la température mesurée sur le sel est probablement excessive par rapport à la réalité; en effet, les palpeurs fixés à la sonde ont sûrement conduit de la chaleur de la sonde (très chaude, $350-400^{\circ} \mathrm{C}$ ) vers le sel (moins chaud, $220^{\circ} \mathrm{C}$ ) ; la valeur «mesurée », vers $280^{\circ} \mathrm{C}$, est donc à considérer comme un majorant de la réalité. Cette réserve faite, on constate une fois de plus l'accord satisfaisant entre les calculs de la température à la paroi du forage (sur le sel); à noter que les calculs de la température de la sonde elle-même sont plus dispersés. Enfin, l'échaufferment du sel se limite à une zone restreinte $\left(1^{\circ} \mathrm{C}\right.$ à $5 \mathrm{~m}$ de l'axe du forage).

\subsubsection{Calculs mécaniques}

De nouveau à titre d'illustration d'un vaste ensemble de résultats, on donne en figure 7 les premières « prévisions * de la convergence, accélérée par le chauffage, de la paroi du forage vers le milieu de la source chaude. Il est clair que le problème fait cette fois intervenir l'activation du fluage par l'intermédiaire de la température élevée; l'analyse mécanique combine donc les difficultés de la convergence libre (IFC) et des contraintes thermiquement induites. Les calculs «encadrent " la valeur expérimentale de la convergence, et respectent l'allure générale de son évolution dans le temps (phase de refroidissement non considérée, comme expliqué plus haut). L'examen détaillé des résultats a montré l'importance primordiale du champ de température, des grandeurs thermoélastiques (module d'Young), de la loi de fluage, sur la valeur de la convergence; la valeur de la pression lithostatique jouerait un rôle plus faible.

\subsection{Résumé des principales observations résultant des calculs}

Des dizaines de calculs rapidement survolés ci-dessus (prévisions initiales et variantes), se dégagent les deux conditions indispensables si l'on veut reproduire par le calcul (ou mieux, prédire) le comportement thermomécanique de structures dans le sel:

- connaître l'état de contrainte initial dans le massif. avant excavation (aspect vital pour le comportement à température ambiante) ;

- disposer d'un modèle rhéologique approprié pour le sel en question.

Pour autant que les codes de calcul soient utilisés par des spécialistes compétents (ce qui fut le cas ici), les aspects purement " numériques * tels que taille et espèce des éléments finis, schémas d'intégration, etc., semblent jouer un rôle moins important quant à la qualité finale des résultats.

\section{CONCLUSIONS ET PERSPECTIVES}

Les paragraphes ci-dessous concernent essentiellement COSA II, mais peuvent se généraliser sans difficultés à l'ensemble du projet COSA.

\subsection{Aspects techniques}

Il convient de noter ici, pour n'y plus revenir, l'indiscutable "valeur ajoutée " par COSA II au programme expérimental relatif au a forage néerlandais » de la mine de Asse ; les premières interprétations et modélisations réalisées par ECN ont indubitablement bénéficié du supplément de recherches apporté par les autres participants.

Un autre point positif fut la nette diminution, dans COSA II par rapport à COSA I, des « erreurs humaines * lors du processus de calcul : entrée des données, présentation et collecte des résultats, etc. Ceci prouve à l'évidence qu'une amélioration de la qualité, dans ce domaine, est possible et se révèle fructueuse. Dans ces conditions, l'adéquation des outils de calcul, quant à leurs aspects « mathématiques » (c'est-à-dire analyse numérique, algorithmes de solution, etc.) a été clairement établie, ainsi d'ailleurs que laptitude et la compétence de leurs utilisateurs. 


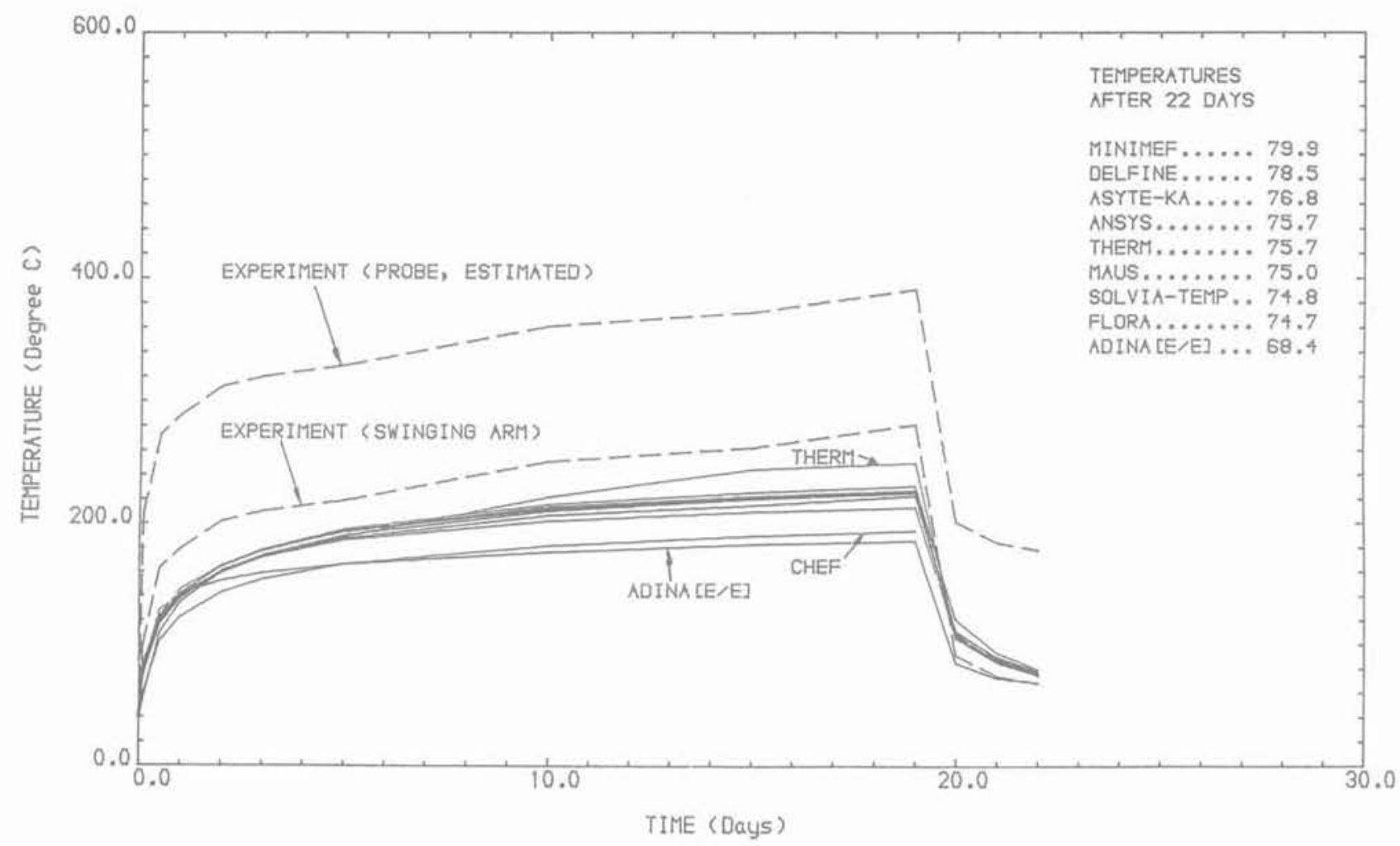

Fig. 6. - Essai «HFCP». Température à la paroi du forage : première comparaison de la mesure (trait interrompu) et des calculs (traits pleins). La courbe supérieure en tireté est la valeur estimée de la température sur la sonde elle-méme.

Fig. 6. - "HFCP and calculations (solid lines). The upper dashed curve is the estimated value of the temperature on the probe itself.

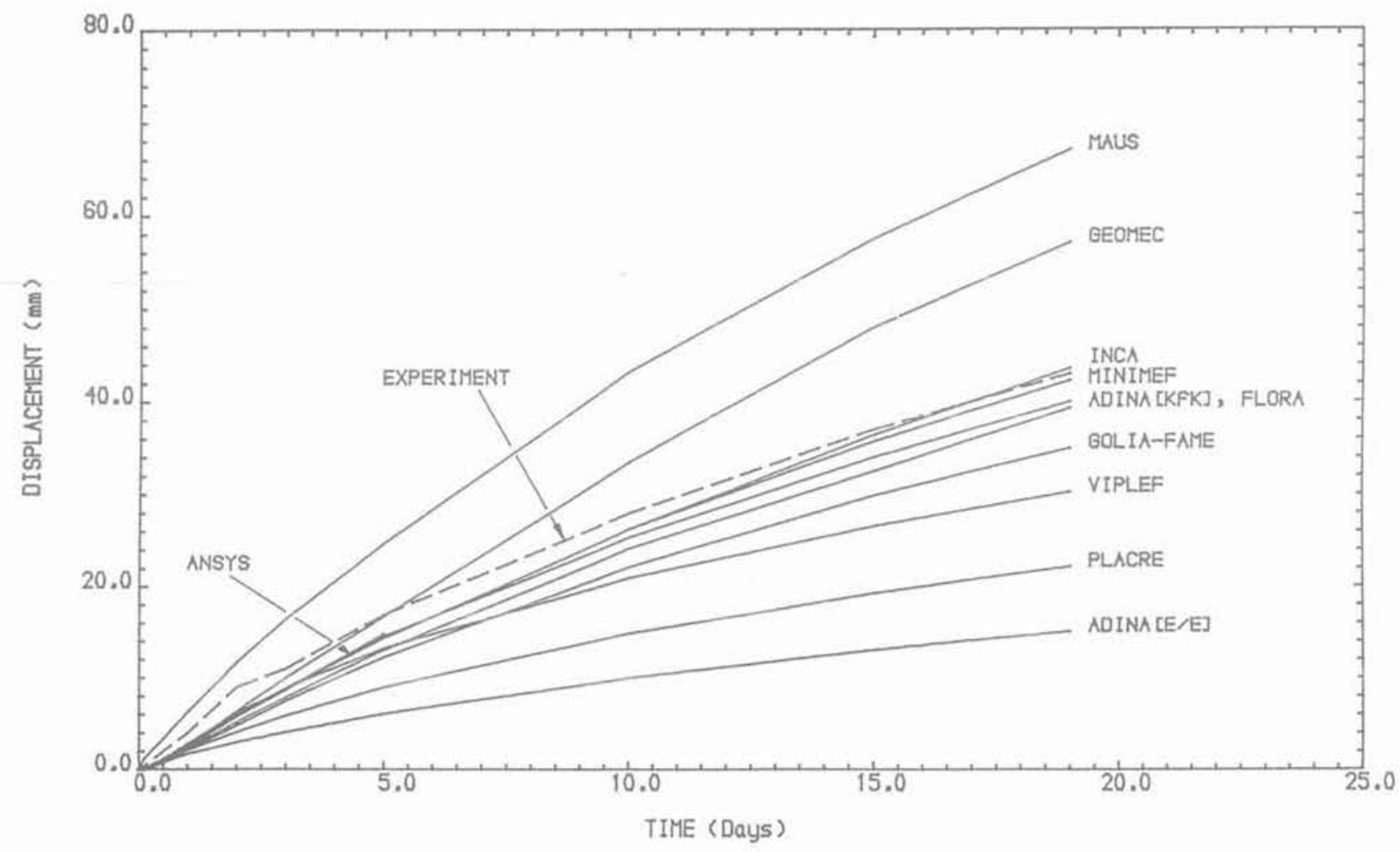

Fig. 7. - Essai « HFCP ». Convergence radiale de la paroi du forage : première comparaison de la mesure (trait interrompu) et des calculs (traits pleins).

Fig. 7. - "HFCP „experiment. Radial convergence of borehole wall: first comparison between measurement ldashed linel and calculations (solid lines). 
L'aspect peut-être le moins satisfaisant, émergeant de COSA II (et de COSA tout entier) serait donc finalement le manque de modèle rhéologique adéquat pour le sel de Asse, manque responsable de la quasiimpossibilité actuelle d'établir de véritables prévisions fiables du comportement thermo-mécanique du sel gemme, par opposition à l'adaptation a posteriori de paramètres via le re-calcul de résultats d'essais connus à l'avance (le "curve-fitting " des Anglo-Saxons). A titre de a consolation $»$, on peut remarquer l'universalité actuelle de cette difficulté, également rencontrée par les spécialistes impliqués dans la construction de l'installation WIPP (Waste Isolation Pilot Plant) au Nouveau-Mexique, USA (MATALUCCI, 1989).

Il s'agit maintenant de replacer ce problème dans le contexte global de l'évaluation de la sûreté d'un dépôt de déchets radioactifs dans le sel, cadre indispensable de projets tels COSA. Il est certain que des itérations sont possibles, entre la conception d'un modèle rhéologique pour un sel (à partir d'essais de laboratoire sur échantillons), sa mise à l'épreuve par confrontation à des essais en place, et son éventuelle modification; mais ceci ne peut bien évidemment résoudre que les aspects " à court et à moyen terme " du problème. C'est le cas du vaste programme de démonstration * HAW * dans la mine de Asse, réalisé par la GSF, I'ECN, l'ANDRA (F) et l'ENRESA (E) avec la participation de la Commission (ROTHFUCHS et al., 1988); pendant les cing années où des conteneurs de verre chargé en radioéléments réels seront insérés, de façon récupérable, dans des forages spécialement équipés, il sera possible de mesurer le comportement thermo-mécanique du sel avoisinant, en particulier la pression exercée par le sel, et de comparer ces mesures à des calculs effectués en parallèle. Là où des prévisions a priori seront nécessaires, et où seuls des calculs pourront être utilisés sans espoir de validation directe, ce sera pour les aspects intimement liés à la sûreté à long terme d'un dépôt dans le sel, par exemple pour calculer le temps que mettront des galeries remblayées de sel broyé à se refermer sur ce dernier, garantissant ainsi une perméabilité extrêmement faible pour le remblayage (CCE, 1988) ; ou bien encore, à beaucoup plus long terme, pour calculer quel sera le comportement thermomécanique global d'un dôme de sel où on aura ins. tallé un dépôt de déchets, pendant la montée en température et pendant le refroidissement ultérieur, et l'influence de ce * cycle sur les terrains avoisinant le dôme. Il est probable d'ailleurs qu'à cette échelle de temps, des mécanismes spécifiques devront être considérés en plus du fluage « traditionnel * (SPIERS, 1988). La nécessité de recherches ultérieures à caractère fondamental sur la rhéologie du sel gemme, combinant judicieusement physique du solide (théorie), essais soigneux au laboratoire sur échantillons et essais en place, apparait donc comme une conclusion incontournable du projet COSA ; elle figurera en bonne place dans les préoccupations du quatrième programme communautaire en matière de déchets radioactifs (1990-1994).

\subsection{Aspects qualitatifs}

Il est également souhaitable, à ce stade, de rappeler quels avaient été, à l'échelle communautaire, les objectifs généraux du projet COSA, initiés dans la première phase et encore amplifiés dans la seconde:

- évaluer la précision numérique et la fiabilité des codes de calculs géomécaniques ;

- évaluer leur facilité d'emploi ( user-friendliness ») ;

- mettre au point une procédure adéquate pour les exercices d'intercomparaison de codes ;

- mettre en lumière les domaines dignes de recherches ultérieures;

- promouvoir l'échange et la mise en commun d'expériences et de connaissances.

Les objectifs qualitatifs ont été, de l'avis unanime des participants, largement atteints, comme a tenté de le montrer ce bref panorama de COSA II ; en particulier, la création d'une véritable communauté de modélisateurs en géomécanique, plus conscients de leurs capacités réelles, n'est probablement pas le moindre résultat de ce projet.

\section{REMERCIEMENTS}

L'auteur tient, une fois encore, à remercier tous les participants au projet COSA (I et II), dont les efforts et l'enthousiasme ont contribué au succès de l'exercice.

\section{BIBLIOGRAPHIE}

CCE, Ouvrage collectif (1988), PAGIS (Performance Assessment of Geological Isolation Systems for Radioactive Waste). Summary Report. Rapport CCE No EUR 11775.

CÔME B. (1987), Le projet communautaire COSA : un exemple d'intercomparaison de codes de calcul géomécaniques pour le sel. Revue Française de Géotechnique, n 40, pp. 23-31.

LOWE M.J.S., KNOWLES N.C. (1986), The Community project COSA : comparison of geomechanical computer codes for salt. Rapport CCE No EUR 10760, Luxembourg. et addendum, rapport $n^{\circ}$ EUR 12134, Luxembourg.

LOWE M.J.S., KNOWLES N.C. (1989), COSA II : Further benchmark exercises to compare geomechanical computer codes for salt. Rapport CCE $n^{\circ}$ EUR 12135, Luxembourg.

MATALUCCI R. (1989), The in-situ testing program at the Waste Isolation Pilot Plant. A paraitre dans * Radioactive Waste Management and the Nuclear Fuel Cycle».

PRIJ J. et al. (1986), Measurements in the 300 metre deep dry-drilled borehole and feasibility study on the dry-drilling of a 600 metre deep borehole in the Asse II salt mine. Rapport CCE $n^{\circ}$ EUR 10737, Luxembourg.

ROTHFUCHS T., DUIJVES K., STIPPLER R. (1988), Das HAW-Projekt: Demonstrationseinlagerung hochradioaktiver Abfälle im Salzbergwerk Asse. Rapport CCE n EUR 11875, Luxembourg.

SPIERS C.J. (1988), Long-term rheological and transport properties of dry and wet salt rocks. Rapport CCE $n^{\circ}$ EUR 11848, Luxembourg. 